\title{
Skin toxicity in BRAF(V600) mutated metastatic cutaneous melanoma patients treated with vemurafenib
}

\author{
Elzbieta Nowara' ${ }^{1}$ Joanna Huszno ${ }^{1}$, Grzegorz Slomian², Jaroslaw Nieckula ${ }^{1}$ \\ ${ }^{1}$ Clinical and Experimental Oncology Department, Cancer Center and Institute of Oncology, Gliwice Branch, Gliwice, Poland \\ ${ }^{2}$ Oncological Ward, Independent Public Health Care Unit, Voivodeship Specialized Hospital, Rybnik, Poland
}

Adv Dermatol Allergol 2016; XXXIII (1): 52-56

DOI: $10.5114 / p d i a .2015 .48045$

\begin{abstract}
Introduction: The use of orally available BRAF kinase inhibitor - vemurafenib is associated with numerous adverse skin reactions.

Aim: To assess the safety and early side effects of vemurafenib treatment in the unselected group of patients treated at the outpatient clinic, in particular the assessment of the incidence of skin cancer.

Material and methods: We carried out a systematic study of patients (pts) treated with vemurafenib. Skin toxicity during vemurafenib therapy was analyzed. Toxicity was determined on the basis of the toxicity scale CTCAE, version 4.0 .

Results: The most common cutaneous side effects were hyperkeratotic perifollicular rash (69\%) and photosensitivity (15\%). Skin rash developed more frequently in the first month of treatment. Squamous cell carcinoma occurred in $38 \%$ of patients. Patients with skin cancer development during vemurafenib therapy had non-significantly longer overall survival (OS) than patients without skin cancer, $p=0.4$. Skin cancer developed more often in women than in men ( $60 \%$ vs. $25 \%), p=0.249$. It was detected only in patients with normal weight compared to overweight patients ( $55 \%$ vs. 0 ), $p=0.09$. The median OS was 26 months and median OS from the time of distant metastases diagnosis was 9.8 months. In patients with a low body mass index, shorter OS was observed, $p=0.09$.

Conclusions: The incidence of squamous cell carcinoma was high (38\%). This study has many limitations mostly due to a small group of patients. That is why the results should be taken into consideration with caution. The proper symptomatic treatment in cooperation with dermatologists allows to continue the vemurafenib therapy.
\end{abstract}

Key words: cutaneous melanoma, BRAF inhibitor, vemurafenib, skin toxicity.

\section{Introduction}

Treatment of metastatic cutaneous melanoma (CM) patients is still a challenge for medicine due to poor prognosis. Median overall survival time (OS) for patients with metastatic CM is 6-15 months after diagnosis [1]. Dacarbazine and interferon, which have been available so far, did not give a high percentage of survival improvement. The response rate is $7-15 \%$ with a median duration of response of 4 months [2]. Multidrug chemotherapy did not significantly prolong OS.

The incidence of CM diagnosis increases. In 2011, in Poland 1259 deaths from CM were reported [3].

The introduction to melanoma treatment molecular targeted therapy during the last year increased the hopes of patients and physicians to improve treatment results. Ipilimumab is a monoclonal antibody against CTLA-4 lym- phocyte receptor. Its mechanism of action interferes with the process of antigen presentation. The final results of this action are increased immune activation, allowing the immune system to recognize, home to, and attack malignant cells. I pilimumab significantly prolongs overall survival of 3.5 months $[4,5]$. Other drugs like dabrafenib or trametenib also showed activity in some patients with malignant melanoma [6].

Activating BRAF kinase mutations occur in $7 \%$ of malignancies and approximately $40-60 \%$ of cutaneous melanoma carry the mutation in BRAF. Vemurafenib demonstrated an unprecedented 60\% antitumor response rate among the patients with BRAF V600 mutated CM. In Poland, until now, only vemurafenib is available to patients with the presence of a mutation in the BRAF V 600 gene. The first refunding possibility has been available since

Address for correspondence: Elzbieta Nowara PhD, Clinical and Experimental Oncology Department, Cancer Center and Institute of Oncology, Gliwice Branch, 15 Wybrzeże Armii Krajowej St, 44-100 Gliwice, Poland, phone: +48 32278 87 17, fax: +48 3227887 16, e-mail: enowara@aol.pl

Received: 6.06.2014, accepted: 3.09.2014. 
July 2013. Due to the short time of patients recruitment, diseases rareness and unusual side effects of this kind of treatment we collected a group of patients treated in two oncology centers.

Vemurafenib treatment is associated with significant and sometimes severe and intriguing treatment-related dermatological toxicity. The most common adverse skin reactions include photosensitivity, induced malignant lesions of the skin such as keratoacanthoma, squamous cell carcinoma and new primary melanomas, as well as keratinocyte proliferation and differentiation dysfunctions that can manifest as skin papillomas, hand-foot skin reaction, keratosis pilaris-like rash, acantholytic dyskeratosis and cysts of the milia type [7-10].

\section{Aim}

The aim of this study was to assess the safety and early side effects of vemurafenib treatment in the unselected group of patients treated at the outpatient clinic, in particular the assessment of the incidence of skin cancer.

\section{Material and methods}

This retrospective analysis was performed at the Clinical and Experimental Oncology Department, Maria Sklodowska-Curie Memorial Cancer Center and Institute of Oncology in Gliwice, Poland (COI). The analysis of patients' medical records was performed according to national laws. The data including the age at onset, disease stage, surgical procedures, and histology were gathered from hospital records and pathology reports. All patients with metastatic or inoperable CM were diagnosed, treated and followed up in COI and in the Oncological Ward of the Independent Public Health Care Unit, Voivodeship Specialized Hospital in Rybnik. All of the patients were treated with the same inclusion criteria including age above 18 years, metastatic or inoperable skin melanoma with a BRAF V600 mutation. All mutations were diagnosed with the same test in the same laboratory. All patients underwent physical, including dermatological, examination and electrocardiography. All patients had a good performance status (ZUBROD 0-1). All patients were evaluated with RECIST 1.1 criteria using computed tomography or magnetic resonance of the brain, chest, abdomen and pelvis. Toxicity was determined on the basis of the toxicity scale CTCAE, version 4.0. All of patients were examined at the outpatient clinic every week. This enabled early toxicity diagnosis and its therapy.

Thirteen consecutive patients were included to the vemurafenib therapy (5 females and 8 males) according to inclusion criteria set by the Polish Ministry of Health. Patients received oral vemurafenib $960 \mathrm{mg}$ twice a day until progression, intolerable adverse effects or withdrawal of consent. Continuation the vemurafenib therapy after disease progression was not allowed. Development of skin carcinoma and its surgical treatment did not require any dose modification or discontinuation of drug.

\section{Statistical analysis}

Statistical analysis was performed using Statistica 8 program. The impact of variables such as patients' gender, previous treatment, response to vemurafenib therapy and toxicity were analyzed by $\chi^{2}$ test with Yates' correction. Differences were considered as significant if the $p$-value was $\leq 0.05$. Survival evaluation was performed using the Kaplan-Meier estimate with a log rank test.

\section{Results}

Median age at the time of CM diagnosis was 54 years (range: 42-70). Nine patients underwent radical surgery and 4 of them received adjuvant radiotherapy. Four (31\%) patients had initial metastatic disease. At the time of inclusion to vemurafenib therapy, all of patients had distant inoperable metastases. The most frequent metastatic sites were mediastinal lymph nodes (in 10 patients - $77 \%$ ), then lungs or liver as well as brain or skin, 39\% and $31 \%$ of patients respectively. Brain metastases were stable and did not require any therapy during vemurafenib intake. Vemurafenib was used in the first- and second-line treatment in 11 (85\%) and 2 (15\%) patients, respectively. Median treatment time was 4.5 months. In some patients we noticed surprisingly quick tumor regressions. The median time of best response was 7 weeks. Disease response (complete response + partial response) was observed in 10 (77\%) patients and disease stagnation (SD) was reached 1 (7\%) patient. During the observation time, 5 patients died. Three of them had disease dissemination to the brain.

During the vemurafenib therapy, all patients developed skin toxicity. In the studied group the most common skin side effects were a diffuse hyperkeratotic perifollicular rash (69\%), photosensitivity (15\%) (Figure 1) and keratoacanthoma (Figure 2). Skin rash developed more

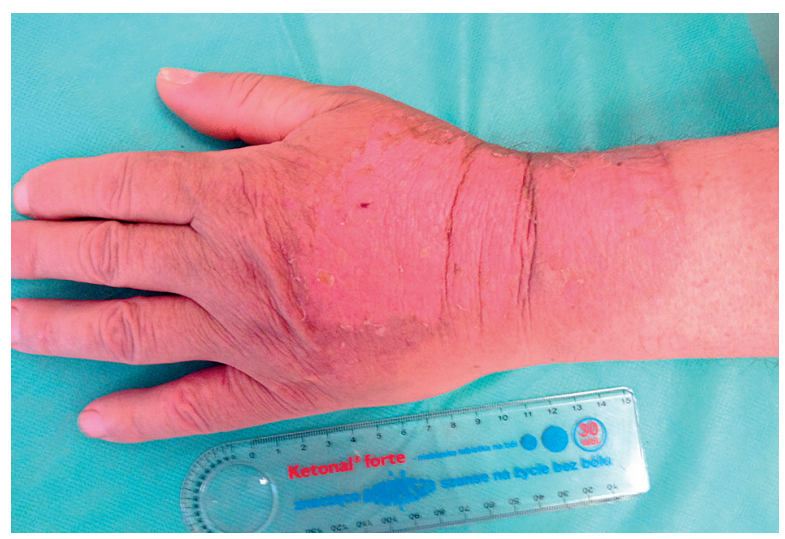

Figure 1. Photosensitivity 

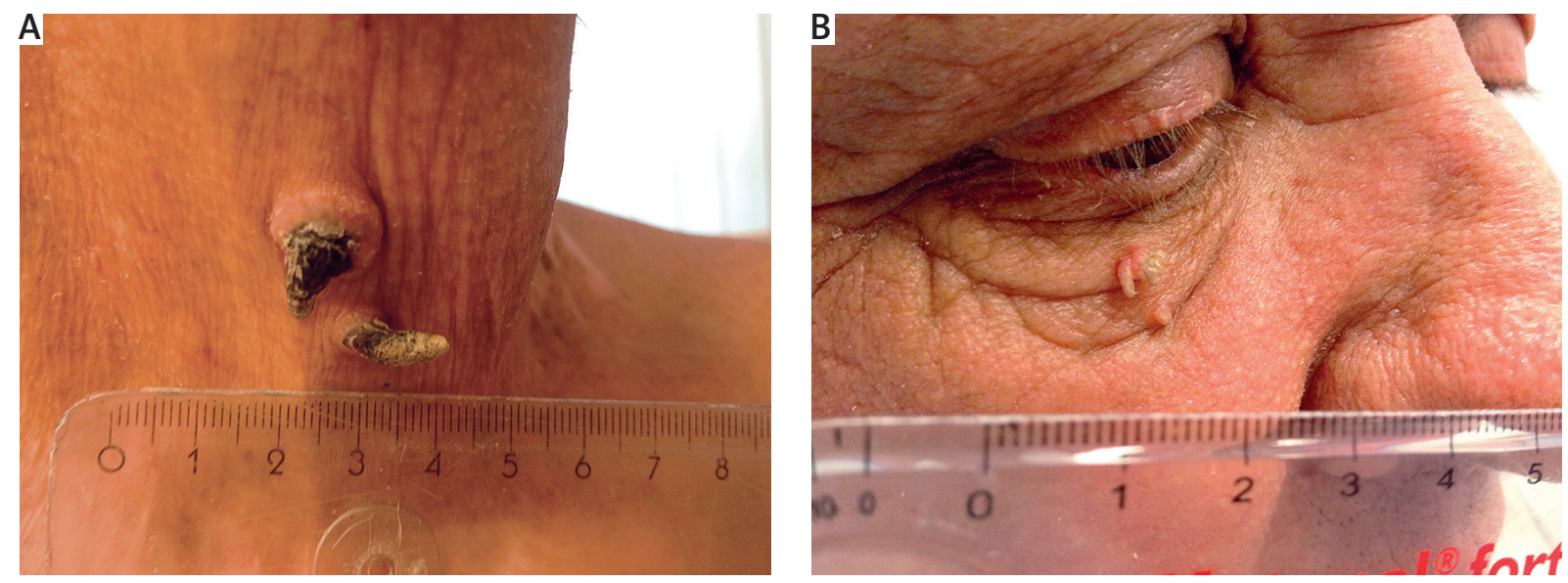

Figure 2. Keratoacanthoma

frequently during the first month of treatment. After symptomatic dermatological treatment (ointments with steroids, antibiotics), lesions have been reduced. Squamous cell carcinoma (SCC) requiring surgery occurred in $38 \%$ of patients. In all patients, skin cancer was located on the face. Surgery did not have any influence on systemic therapy administration. Median time to skin cancer development was 8 weeks.

Skin cancer developed more often in women than in men (60\% vs. $25 \%), p=0.249$. It was detected only in patients with normal weight compared to overweight patients ( $55 \%$ vs. 0\%), $p=0.09$. Patients with skin cancer developed during vemurafenib therapy had non-significantly longer OS than patients without skin cancer (11.8 vs. 9.4 months), $p=0.4$. Other common toxic effects were severe musculoskeletal pain (in 8 patients) and high fever (in 5 patients).

Median body mass index (BMI) was 26.6 (range: 2239) $\mathrm{kg} / \mathrm{m}^{2}$. Two patients had normal weight, and 7 were overweight and 4 were obese. Body mass index had no influence on the skin toxicity incidence. Patients with low BMI had non-significantly shorter OS. Ten (77\%) patients had comorbid conditions such as hypertension, cardiovascular diseases and other in history.

Treatment response was observed more frequently in men than in women. The differences were not significant $(p=0.128)$. The better response was detected in patients who received vemurafenib in the first line of treatment $(p=0.294)$. Vemurafenib toxicity and treatment response are summarized in Table 1.

The median overall survival (OS) was 26 months and median OS from the time of distant metastases diagnosis was 9.8 months. Patient overall survival is summarized in Table 2.

\section{Discussion}

This retrospective study of early vemurafenib toxicity showed that all patients developed side effects related to vemurafenib therapy. Skin lesions occurred in the first month of treatment. The most common skin side effects were a diffuse hyperkeratotic perifollicular rash (69\%) and photosensitivity (15\%). The management and evolution of the skin symptoms were described by Bous-

Table 1. Vemurafenib toxicity and treatment response

\begin{tabular}{|c|c|c|}
\hline Parameter & Percentage of patients & Statistical significance, $p$ \\
\hline \multicolumn{2}{|c|}{ Skin cancer development during treatment: } & \multirow{3}{*}{0.249} \\
\hline Women & 60 & \\
\hline Men & 25 & \\
\hline \multicolumn{2}{|c|}{ Skin cancer development during treatment: } & \multirow{3}{*}{0.09} \\
\hline Overweight patients & 55 & \\
\hline Patients with normal weight & 0 & \\
\hline \multicolumn{2}{|l|}{ Treatment response: } & \multirow{3}{*}{0.128} \\
\hline Women & 40 & \\
\hline Men & 95 & \\
\hline
\end{tabular}


Table 2. Patients overall survival

\begin{tabular}{lcc}
\hline Parameter & Time [months] & Statistical significance, $p$ \\
\hline Median overall survival: & & \\
\hline Women & 13.7 & 0.3 \\
\hline Men & 36.0 & \\
\hline Median overall survival for patients: & 11.8 & 0.4 \\
\hline With skin cancer development & 9.4 & \\
\hline Without skin cancer development & & \\
\hline
\end{tabular}

semart et al. [11]. In this study most common cutaneous toxicity was verrucous papillomas (79\%), perifollicular rash (55\%), photosensitivity (52\%) and alopecia (45\%). Keratoacanthoma (KA) occurred in 14\% and squamous cell carcinoma (SCC) in $26 \%$ of the patients [12]. The adverse events were consistent with toxicity reported in the BRIM-2 and BRIM-3 registration trials [10-12]. In BRIM-3 trial, the most frequent grade 3-4 events were cutaneous squamous-cell carcinoma (19\% of patients) and keratoacanthoma (10\%), rash (9\%), and abnormal liver function tests (11\%) in the vemurafenib group [12]. In the analyzed group, SCC occurred in $38 \%$ of patients. The incidence of cutaneous squamous cell carcinoma was higher than that reported in BRIM-2 and BRIM-3 (26\% and 19\%, respectively). The mechanism of skin cancer development during vemurafenib therapy is still under investigation. Neoplasms originating from keratocytes were observed during therapy with other BRAF inhibitor - dabrafenib and multikinase inhibitor - sorafenib.

Authors have attempted to find the influence of clinicopathological factors on skin toxicity and skin cancer development during vemurafenib therapy. Skin cancer developed slightly more often in women than in men $(p=0.249)$ and in patients with normal weight compared to overweight patients $(p=0.09)$. Authors did not find any data on this point in the literature and do not have any explanation for this. There was no association between patients' age, comorbid conditions and skin toxicity of vemurafenib. In an open label, multicentre trial described by Larkin et al., there was an increased incidence of grade 3 and grade 4 adverse events, and adverse events leading to discontinuation in patients aged 75 years and older [9]. Other side effects was QTC prolongation. In this trial, cardiac comorbidity was a risk factor for QTc prolongation and long-term sun damage to the skin for the skin cancers [9]. We did not observe QTC prolongation in the studied group.

Vemurafenib produced improved rates of overall and progression-free survival in patients with previously untreated melanoma with the BRAF V600E mutation [12]. Median PFS and overall survival were 5.6 months and 12.0 months, respectively [6]. Similar overall survival was noted in the pivotal phase 2 and 3 trials $[13,14]$. In the analyzed group, the median overall survival (OS) was
26 months and median OS from the time of distant metastases diagnosis was 9.8 months. In patients with lower BMI shorter OS was observed.

\section{Conclusions}

All of the patients developed skin toxicity. The incidence of squamous cell carcinoma was high (38\%). The results of this study has many limitations mostly due to a small group of patients. That is why the results should be taken into consideration with caution. The proper symptomatic treatment in cooperation with dermatologists allows to continue the vemurafenib therapy.

\section{Conflict of interest}

The authors declare no conflict of interest.

\section{References}

1. Manola J, Atkins M, Ibrahim J, et al. Prognostic factors in metastatic melanoma: a pooled analysis of Eastern Cooperative Oncology Group trials. J Clin Oncol 2000; 18: 3782-93.

2. Onkologia w Praktyce Klinicznej. Zalecenia postępowania diagnostyczno-terapeutycznego w nowotworach złośliwych 2013 rok.

3. http://epid.coi.waw.pl/krn/

4. Graziani G, Tentori L, Navarra P. Ipilimumab: a novel immunostimulatory monoclonal antibody for the treatment of cancer. Pharmacol Res 2012; 65: 9-22.

5. Traynor K. Ipilimumab approved for metastatic melanoma. Am J Health Syst Pharm 2011; 68: 768.

6. Hauschild A, Grob JJ, Demidov LV, et al. Dabrafenib in BRAFmutated metastatic melanoma: a multicentre, open-label, phase 3 randomised controlled trial. Lancet 2012; 380: 35865.

7. Wantz M, Spanoudi-Kitrimi I, Lasek A, et al. Vemurafenibinduced toxic epidermal necrolysis. Ann Dermatol Venereol 2014; 141: 215-8.

8. Hersey P. Community experience of vemurafenib for BRAF(V600) melanoma. Lancet Oncol 2014; 15: 369-70.

9. Larkin J, Del Vecchio M, Ascierto PA, et al. Vemurafenib in patients with BRAF(V600) mutated metastatic melanoma: an open-label, multicentre, safety study. Lancet Oncol 2014; 15: 436-44.

10. Huang V, Hepper D, Anadkat M, et al. Cutaneous toxic effects associated with vemurafenib and inhibition of the BRAF pathway. Arch Dermatol 2012; 148: 628-33. 
11. Boussemart L, Routier E, Mateus C, et al. Prospective study of cutaneous side-effects associated with the BRAF inhibitor vemurafenib: a study of 42 patients. Ann Oncol 2013; 13: 1-7.

12. McArthur GA, Chapman PB, Robert C, et al. Safety and efficacy of vemurafenib in BRAFV600E and BRAFV600K mutation-positive melanoma (BRIM-3): extended follow-up of a phase 3, randomised, open-label study. Lancet Oncol 2014; 15: 323-32.

13. Sosman JA, Kim KB, Schuchter L, et al. Survival in BRAF V600-mutant advanced melanoma treated with vemurafenib. N Engl J Med 2012; 366: 707-14.

14. Chapman PB, Hauschild A, Robert C, et al. Improved survival with vemurafenib in melanoma with BRAF V600E mutation. N Engl J Med 2011; 364: 2507-16. 